\title{
Population kinetics during simultaneous infection of insect cells with two different recombinant baculoviruses for the production of rotavirus-like particles
}

\author{
Jimmy A Mena, Octavio T Ramírez and Laura A Palomares*
}

Address: Departamento de Medicina Molecular y Bioprocesos, Instituto de Biotecnología, Universidad Nacional Autónoma de México (UNAM), Apdo. Postal. 510-3. Cuernavaca, Morelos, CP. 62250, México

Email: Jimmy A Mena - jimmy@ibt.unam.mx; Octavio T Ramírez - tonatiuh@ibt.unam.mx; Laura A Palomares* - laura@ibt.unam.mx

* Corresponding author

Published: 4 July 2007

BMC Biotechnology 2007, 7:39 doi:10.1186/1472-6750-7-39
Received: 22 February 2007

Accepted: 4 July 2007

This article is available from: http://www.biomedcentral.com/1472-6750/7/39

(c) 2007 Mena et al; licensee BioMed Central Ltd.

This is an Open Access article distributed under the terms of the Creative Commons Attribution License (http://creativecommons.org/licenses/by/2.0), which permits unrestricted use, distribution, and reproduction in any medium, provided the original work is properly cited.

\begin{abstract}
Background: The simultaneous production of various recombinant proteins in every cell of a culture is often needed for the production of virus-like particles (VLP) or vectors for gene therapy. A common approach for such a purpose is the coinfection of insect cell cultures with different recombinant baculoviruses, each containing one or more recombinant genes. However, scarce information exists regarding kinetics during multiple infections, and to our knowledge, no studies are available on the behavior of the different populations that arise during coinfections. Such information is useful for designing infection strategies that maximize VLP or vector yield. In this work, kinetics of cell populations expressing rotavirus GFPVP2 (infected with bacGFPVP2), VP6 (infected with bacVP6), or both proteins simultaneously (coinfected with both baculoviruses) were followed by flow cytometry.
\end{abstract}

Results: In single infections, the population infected with any of the recombinant baculoviruses followed the Poisson distribution, as the population expressing a recombinant protein exhibited a hyperbolic-type function with respect to the multiplicity of infection (MOI) up to 5 pfu/cell. In coinfections, the population fraction expressing each recombinant protein could not be anticipated from results of single infections, as in some cases interference and synergistic effects were found. Only cultures with a total $\mathrm{MOI}$ below 5 pfu/cell followed the Poisson distribution. For cultures with a MOI of bacGFPVP2 above that of bacVP6 (overall MOI above 5 pfu/cell), the total population expressing one or both recombinant proteins was as low as $50 \%$ below that predicted by Poisson. In contrast, the population fraction expressing VP6 increased in coinfections, compared to that in single infections. The largest population fraction simultaneously expressing both recombinant proteins was $58 \%$, and corresponded to cultures infected at a MOI of 5 and I pfu/cell of bacGFPVP2 and bacVP6, respectively.

Conclusion: The infection conditions that maximize the cell population simultaneously expressing two recombinant proteins were determined. Such conditions could not have been anticipated from population kinetics in individual infections. This information should be taken into account for improved simultaneous production of various recombinant proteins in any work dealing with coinfections. 


\section{Background}

Virus-like particles (VLP) are structurally identical to native viruses, but they lack the viral genetic material [1]. VLP are obtained when the major viral structural proteins are simultaneously expressed in a recombinant system. There exists an increasing interest on VLP production due to their promising applications as vaccines, as delivery vehicles for substances or genes, or as biosensors [1]. A recent example of the importance of VLP is the recent approval of Merck's vaccine against human papilloma virus. The production of VLP is a complex process and a challenging task, as it requires the simultaneous expression of various recombinant proteins. Due to its versatility and simplicity for coexpressing various recombinant genes, the insect-cell baculovirus expression vector system (IC-BEVS) has been commonly employed for producing VLP of several viruses.

The simultaneous production of several proteins in insect cells requires the delivery of various genes, either by a number of individual baculoviruses or by employing a single virus that contains several genes [2-4]. Of these strategies, the use of individual baculoviruses allows the manipulation of the concentration of each protein by changing the multiplicity of infection (MOI) of each virus $[3,5,6]$. In this way, the stoichiometry between the structural proteins may be controlled. In some VLP, which can have variable protein composition, changes in the ratio between structural proteins result in different VLP compositions, which can yield particles with different immunogenicity $[5,6]$. It is also possible that different stoichiometries between the structural proteins result in changes in VLP assembly efficiencies or kinetics, although this remains to be studied. Therefore, MOI manipulation is a powerful tool for finding the conditions required for maximizing the assembly of a desired VLP. However, little is known about the performance of simultaneous infections with various recombinant baculoviruses, specifically regarding cell population kinetics and possible interferences or synergies between the coinfecting viruses.

Rotavirus is a triple-layered virus that is responsible of gastroenteritis. The inner layer, a core-like particle, is constituted by VP2, surrounded by a second concentric layer containing VP6. The third layer is formed by VP7 and spikes of VP4 [7]. Recently, Mena et al. [8] studied the accumulation in insect cells of double layered rotaviruslike particles (dIRLP), that are constituted by the two inner concentric layers. They found that the assembly of dIRLP occurs intracellularly, and that, when expressed individually, both VP2 and VP6 form structures that cannot further assemble into double-layered particles. Namely, under such condition VP6 forms tubes whereas VP2 forms corelike particles densely packed in ordered groups. Neither tubes nor densely packed cores are accessible for interac- tion with the other recombinant protein, and thus dIRLP cannot be formed. These findings underline the importance of having both VP2 and VP6 available for assembly into dIRLP in each cell of the culture and at the right time to avoid the formation of structures composed only by a single protein. A similar situation occurs when other VLP are produced, such as adeno-associated viral vectors, which require the simultaneous expression of the structural and non-structural proteins, as well as replication of vector DNA in the same cell [9]. It can be anticipated that appropriate infection strategies, based on the manipulation of MOI, would maximize VLP yield. Several groups have studied the dynamics of infection at various MOI, while others have predicted by mathematical modeling the percentage of the population infected under different conditions [2-5,10-14]. Nonetheless, cell population kinetics during the simultaneous infection with two recombinant viruses have, to our knowledge, not been studied

A powerful tool for assessing the dynamics of infection at different MOI is flow cytometry. This methodology has been used to determine the percentage of the population infected at different MOI by a single type of virus $[15,16]$, for assessing the productivity of different cell lines [17], to measure respiratory activity [18], to titer viruses [19], and to determine transducing titers of gene delivery vectors [20]. Flow cytometry has also been used to screen BF2 fish cells simultaneously infected with non-recombinant viruses of two different species; the pancreatic necrosis virus and the hematopoietic necrosis virus [21]. In our work, insect cells were infected with one or two different recombinant baculoviruses, expressing rotavirus VP2 or VP6, at different MOI. Utilizing flow cytometry we have, for the first time, determined the kinetics of cell populations expressing either of the recombinant proteins during simultaneous infections with both recombinant baculoviruses and compared them with single infections. Moreover, we have determined the infection conditions required to guarantee that the highest fraction of the population is simultaneously expressing both recombinant proteins, one of the necessary conditions for complete and efficient dlRLP production.

\section{Results \\ Cultures infected with a single baculovirus}

Insect cell cultures individually infected with bacGFPVP2 or VP6 were analyzed by flow cytometry at 24 and 48 hours post-infection (hpi). MOI of $0.1,1,5,10$, and 20 pfu/cell were tested. Fluorescence histograms at 24 hpi are shown in Figure 1, along with an uninfected control culture performed simultaneously. When analyzing these results, it should be taken into account that fluorescence emission was measured at two different wavelengths, 510 for GFPVP2 and $575 \mathrm{~nm}$ for immunolabeled gp64 and 
VP6. Thus, background fluorescence of uninfected cells was different. Cells expressing a recombinant protein could be easily distinguished from uninfected cells. The distribution of the populations expressing GFPVP2 was different from the one expressing VP6. Data extracted from fluorescence histograms of duplicate cultures are summarized in Figure 2A, where the fraction of the population expressing a recombinant protein is reported relative to the total cell count (regardless of viability). It should be considered that the analysis shown in Figure 1 allows the identification of the population expressing a recombinant protein (either GFPVP2 or VP6), but not necessarily that of infected cells, as expression of the recombinant gene might be absent or inefficient even in infected cells. A marker that has been used to monitor infection by baculovirus is gp64 [22]. gp64 is the major envelope glycoprotein of baculovirus [23], and accumulates in the membrane of infected cells during the first 10 to 12 hpi [24]. To correlate infection with recombinant protein expression, gp64 was immunodetected at $24 \mathrm{hpi}$ in cultures expressing GFPVP2 (Figures 1 and 2A). In addition, the population distribution predicted by Poisson:

$$
\mathrm{p}(\mathrm{w})=\left[\left(\frac{\mathrm{MOI}^{\mathrm{w}}}{\mathrm{w} !}\right) e^{-\mathrm{MOI}}\right]
$$

commonly used to describe infection, is also plotted in Figure 2A[3]. The population fraction expressing any of the recombinant proteins or gp64 increased in a hyperbolic-type function with MOI up to $5 \mathrm{pfu} / \mathrm{cell}$, and then remained constant for MOI above $5 \mathrm{pfu} /$ cell. Such a behavior is in agreement with predictions based on the Poisson distribution. Cultures infected with bacVP6 or bacGFPVP2 had a similar trend, which was also similar to the population expressing gp64 in the cultures infected with bacGFPVP2. The similar behavior between cells expressing GFPVP2, gp64 and VP6 confirms that the results obtained from cells immunostained for VP6 were representative of the population expressing that recombinant protein, and that both recombinant baculoviruses were equally infective. Results obtained at $48 \mathrm{hpi}$ are shown in Figure 2B. The Poisson distribution is not plotted in Figure 2B, as secondary infection had occurred at this time, and different calculations are needed to predict the infected population under these conditions (see discussion below). At $48 \mathrm{hpi}$, between 65 to $90 \%$ of the cells were expressing either recombinant protein, regardless of the MOI. However, at MOI below $10 \mathrm{pfu} / \mathrm{cell}$, a smaller population of cells expressed GFPVP2, in comparison with that expressing VP6.

Handling of cultures expressing GFPVP2 for FACS analysis was easier than those for VP6, as VP6 had to be immunostained by a laborious procedure, whereas intrinsic fluores- cence of GFPVP2 facilitated the assay. Therefore the population containing this recombinant protein was followed every $24 \mathrm{~h}$ until 96 hpi. Results are shown in Figure $3 \mathrm{~A}$. The population expressing GFPVP2 reached a maximum at 24 hpi for cultures infected at a MOI of $5 \mathrm{pfu} / \mathrm{cell}$ or higher. In contrast, such population reached a maximum until 48 hpi for cultures infected at MOI of less than $5 \mathrm{pfu} / \mathrm{cell}$. In all cases, the percentage of the population expressing a recombinant protein decreased after $48 \mathrm{hpi}$ (Figure $3 \mathrm{~B}$ ), probably due to degradation of the recombinant protein after loss of cell membrane integrity in non-viable cells. Viability decreased steadily after infection, reaching $0 \%$ at $72 \mathrm{hpi}$ in cultures infected at MOI of 10 and $20 \mathrm{pfu} / \mathrm{cell}$. The rate of decrease of the viability was lower as the MOI decreased.

\section{Cultures simultaneously infected with bacGFPVP2 and bacVP6}

Gating parameters used for discriminating between cells expressing GFPVP2, VP6 or both proteins were set according to fluorescence (emission) at $510 \mathrm{~nm}$ (corresponding to GFP) and $575 \mathrm{~nm}$ (corresponding to R-phycoerythrin, used to detect VP6) in uninfected cultures, cultures expressing only GFPVP2, or cultures expressing VP6. Typical results are shown in Figures 4A-C. As expected, uninfected cultures had a low emission both at 510 or $575 \mathrm{~nm}$ (Figure 4A), whereas cells infected with bacGFPVP2 had a high emission at $510 \mathrm{~nm}$ (Figure 4B). Moreover, cells infected with bacVP6 had a high fluorescence at $575 \mathrm{~nm}$ (Figure 4C). Accordingly, gating parameters, which allowed the differentiation between the three populations present in coinfected cultures, could be set. Flow cytometry results of a culture simultaneously infected with bacGFPVP2 and VP6 at 24 or 48 hpi are shown in Figure $4 \mathrm{D}$ and $4 \mathrm{E}$, respectively. At 24 hpi many cells could be clearly classified as expressing VP6, whereas only relatively few cells were expressing both recombinant proteins. At 48 hpi, only a few cells were not expressing a recombinant protein, while most were either expressing VP6 or both recombinant proteins simultaneously. A small fraction of the population was expressing only GFPVP2. A quantitative analysis of the flow cytometry study is presented below.

The results obtained from 4 experimental setups performed in duplicate are summarized in Figure 5. Cultures were infected at different MOI combinations of bacGFPVP2 and bacVP6. MOI of each virus were not increased above $5 \mathrm{pfu} / \mathrm{cell}$, as higher MOI did not result in a higher population fraction expressing any of the recombinant proteins in single infections (Figure 2). In most cases, the largest fraction of the population expressed VP6, regardless of the MOI of bacGFPVP2. The only exceptions were the cultures infected with 0.1 and $5 \mathrm{pfu} / \mathrm{cell}$ of bacVP6 and bacGFPVP2, respectively, either at 24 or 48 


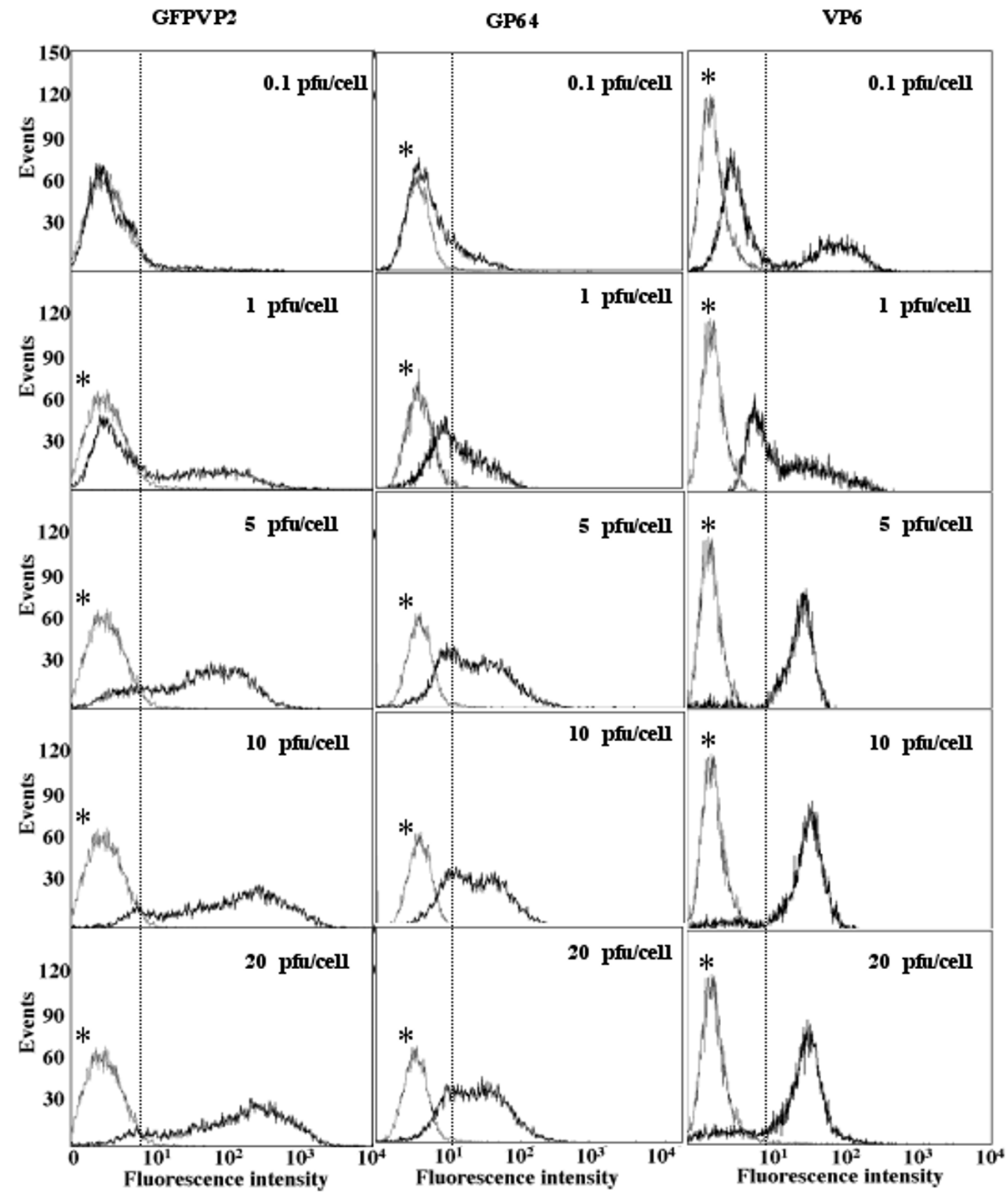

Figure I

Fluorescence histogram analysis of cells infected with bacGFPVP2 or bacVP6 at different multiplicities of infection at 24 hpi. Fluorescence of GFPVP2, immunolabeled gP64 in cells expressing GFPVP2, and immunolabeled VP6 are shown in black. A control-uninfected culture is shown in gray and marked as (*). Dotted vertical line corresponds to the fluorescence gating used. 


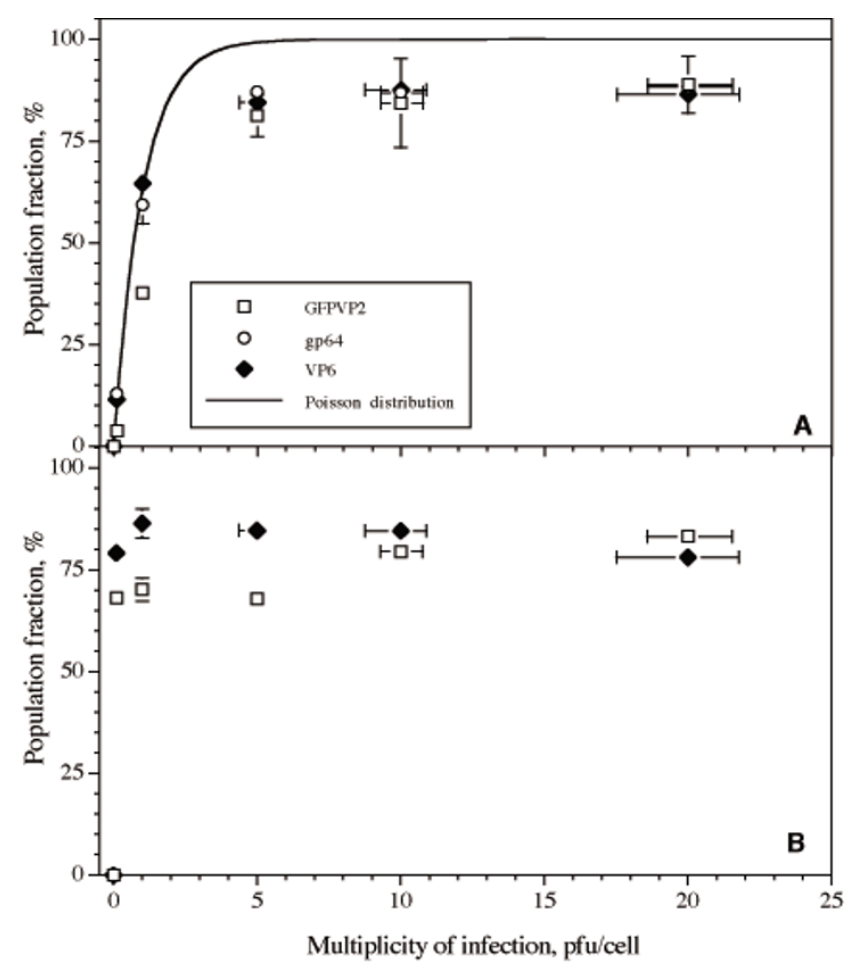

Figure 2

Cultures individually infected with bacGFPVP2 or bacVP6. Both sets of cultures are plotted in the same graphs to allow comparison. A. Fraction of the population expressing GFPVP2, gp64, or VP6 at 24 hours post infection (hpi). The probability $(p)$ of a population of being infected by $w$ virus, predicted by Poisson, is shown as a solid line. $B$. The same cultures as in A but at $48 \mathrm{hpi}$. Media and difference between duplicate cultures are shown in $Y$ axis error bars. $X$ error bars represent the standard deviation of titers of the viral stocks, adjusted to each MOI. Some error bars are smaller than the symbols shown.

hpi. In these cultures, the population expressing either of the recombinant proteins was about the same. It should be noted that, in the extreme situation, the population fraction expressing VP6 was 17.5 times larger than that expressing GFPVP2 (MOI bacVP6 1 pfu/cell, MOI bacGFPVP2 $0.1 \mathrm{pfu} / \mathrm{cell}, 24 \mathrm{hpi}$ ). The highest percentage of the population expressing any recombinant protein (VP6 or GFPVP2 or both) at $24 \mathrm{hpi}$ was obtained at MOI of $1 \mathrm{pfu} /$ cell for both bacGFPVP2 and bacVP6, i.e. a total MOI of $2 \mathrm{pfu} /$ cell. Such population fraction was $15 \%$ higher than that expressing any recombinant protein at the highest total MOI tested, which corresponded to 6 pfu/cell (5 pfu/cell of bacGFPVP2 and 1 pfu/cell of VP6). The population fraction expressing both recombinant proteins at 24 hpi closely followed the population expressing GFPVP2.

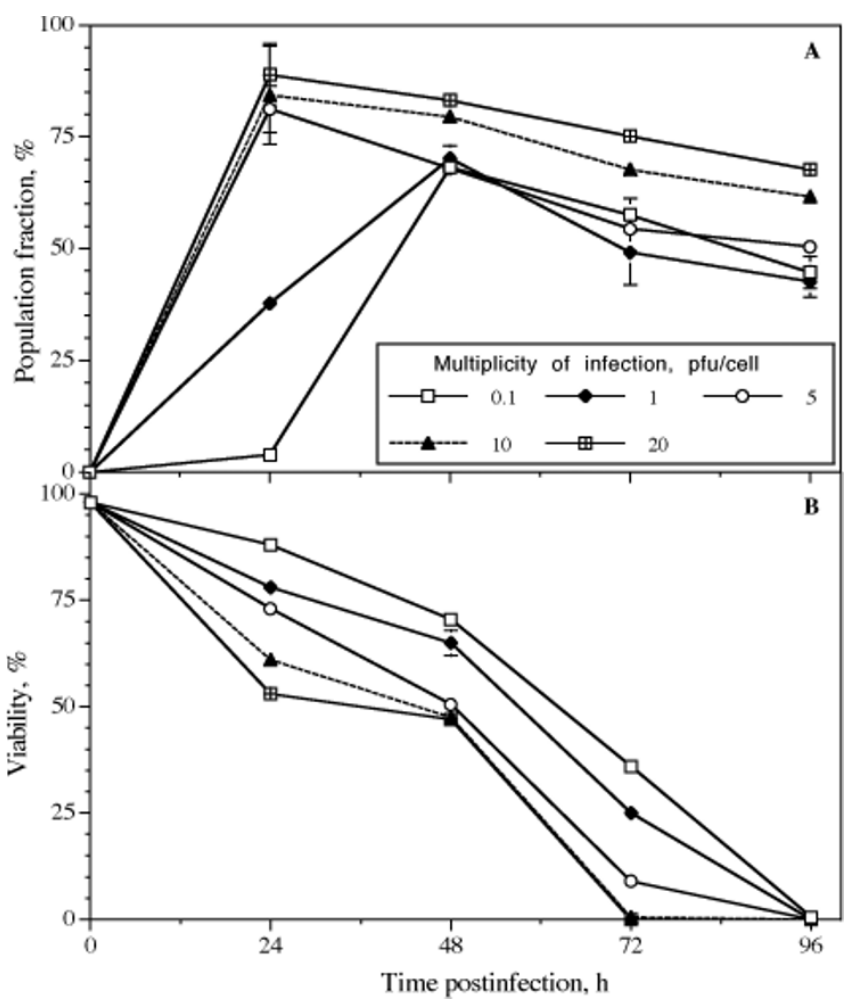

Figure 3

Cultures infected only with bacGFPVP2. A. Kinetics of the fraction of the population expressing GFPVP2 in cultures infected only with bacGFPVP2. Numbers in figure legend refer to the different $\mathrm{MOI}$ used. B. Viability of cultures infected with bacGFPVP2. Media and difference between duplicate cultures are shown. Some error bars are smaller than the symbols shown.

Coinfections had various effects on the population expressing GFPVP2. In general, the cell population expressing GFPVP2 in coinfected cultures was smaller than in single-infected cultures at the same MOI, except in two cases at 24 hpi (Figures 2 and 5). Namely, in cultures infected at a MOI of $0.1 \mathrm{pfu} / \mathrm{cell}$ of each baculovirus, the population expressing GFPVP2 was duplicated, in comparison to that observed in single infections. Moreover, at $0.1 \mathrm{pfu} / \mathrm{cell}$ of bacVP6 and $1 \mathrm{pfu} / \mathrm{cell}$ of bacGFPVP2, the population expressing GFPVP2 was the same than that observed in single infections. In contrast, the population expressing VP6 at 24 hpi increased in coinfections, compared to individual infections. In cultures infected at MOI of $0.1 \mathrm{pfu} / \mathrm{cell}$ of bacVP6, $11.5 \%$ of the population expressed VP6 in single-infected cultures, whereas in coinfections up to $45 \%$ of the population expressed VP6 (Figures 2 and 5). Something similar occurred at a MOI of bacVP6 of 1pfu/cell, as $65 \%$ of the population expressed VP6 in single-infected cultures, while in coinfections such percentage increased to $70-85 \%$ at $24 \mathrm{hpi}$. 

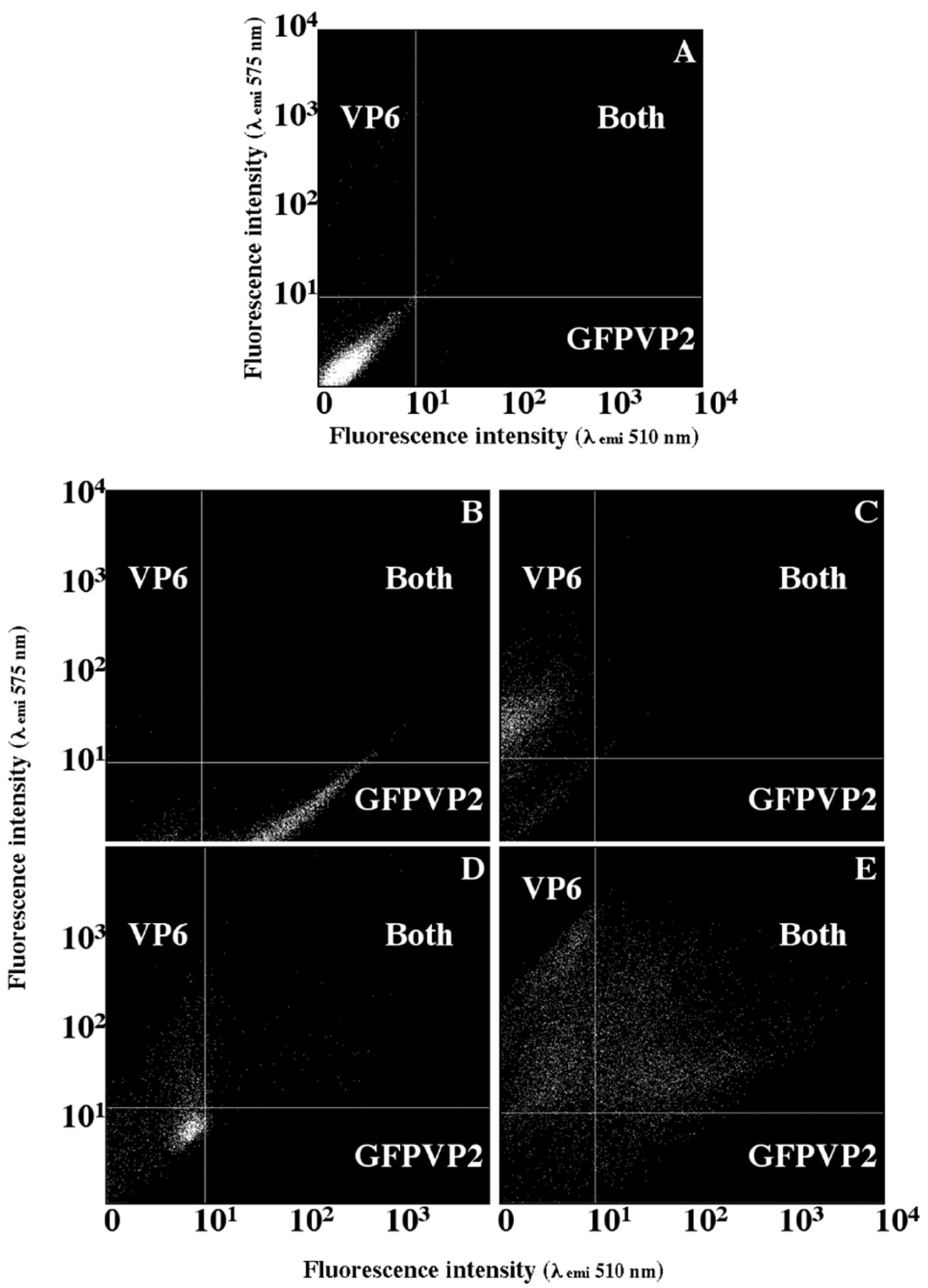

Figure 4

Identification of cell populations simultaneously expressing GFPVP2 and VP6. Cultures shown in panels A to C were used for setting the gating limits for analysis of coinfected cultures. A. Uninfected control culture. B. Culture at 24 hpi infected with bacGFPVP2 at a MOI of 5 pfu/cell. C. Culture at 24 hpi infected with bacVP6 at a MOI of 5 pfu/cell. D. Culture coinfected with bacGFPVP2 and bacVP6 with a MOI of 0.1 pfu/cell of each virus, 24 hpi. E. Culture coinfected with bacGFPVP2 and bacVP6 with a MOI of 0.1 pfu/cell of each virus, $48 \mathrm{hpi}$. 
Compared to $24 \mathrm{hpi}$, the population expressing any of the recombinant protein increased at 48 hpi (Figure 5). This increase occurred even in cultures infected with a MOI of bacGFPVP2 of 5 pfu/cell; a behavior not observed in individual infections. The population fraction expressing VP6 at 48 hpi reached $98 \%$ (considering the error bars) in cultures infected at MOI of 0.1 or $1 \mathrm{pfu} /$ cell of any of the baculoviruses. In most cases the percentage of the population expressing GFPVP2 at 48 hpi was smaller than that expressing VP6, with the exception of the cultures infected with $0.1 \mathrm{pfu} / \mathrm{cell}$ of bacVP6 and $5 \mathrm{pfu} / \mathrm{cell}$ of bacGFPVP2, where the population expressing either protein was equal. The condition that resulted in the highest percentage of the population simultaneously expressing both recombinant proteins was a MOI of bacGFPVP2 of $5 \mathrm{pfu} / \mathrm{cell}$ and $1 \mathrm{pfu} / \mathrm{cell}$ of bacVP6. Such condition resulted in $48 \%$ and $58 \%$ of the population at 24 and $48 \mathrm{hpi}$, respectively, simultaneously expressing both recombinant proteins. Populations expressing the recombinant proteins were not followed after $48 \mathrm{hpi}$ as further infections by progeny viruses were not expected after $48 \mathrm{hpi}$, and a rapid decline in viability was observed after this time (data not shown).

To better appreciate the effect of coinfections, the population expressing any of the recombinant proteins (GFPVP2, VP6, or both) at 24 hpi is shown in Figure 6 as a function of total MOI (MOI bacGFPVP2 + MOI bacVP6). For comparison, the Poisson distribution is also plotted. It can be seen that coinfected cultures with a MOI of bacVP6 equal or higher than that of bacGFPVP2 followed the Poisson distribution. However, cultures where the MOI of bacGFPVP2 was 5,10 , or 50 times higher than that of bacVP6 had a much lower population expressing any of the recombinant proteins than that predicted by the Poisson distribution at the corresponding cumulative MOI.

\section{Discussion}

Previous reports [15-17] and this work demonstrate the utility of flow cytometry for monitoring recombinant protein expression. In this work, a wider screening of recombinant protein expression at different MOI and at different times postinfection was performed, in addition to following the populations expressing two recombinant proteins during coinfections. At $24 \mathrm{hpi}$, the percentage of the population expressing VP6 and gp64 closely followed the Poisson distribution. This observation is relevant, as it has been shown that various culture variables, including medium composition, mode of culture, temperature, etc., can affect infection [25-27]. The percentage of the population expressing a recombinant protein did not reach $100 \%$, most probably due to the viability of cultures at the time of infection, which was between 96 and $98 \%$. It should be noted that the population expressing GFPVP2 at $24 \mathrm{hpi}$ with a MOI below $5 \mathrm{pfu} /$ cell was in most cases lower than that expressing gp64 (detected in the culture infected with bacGFPVP2) or VP6. It is possible that the smaller population expressing GFPVP2 was a result of the slower production rate of this protein in comparison with VP6, as has been observed previously $[3,4,14]$. Thus, in some cells the amount of GFPVP2 at 24 hpi may be below the detection limit of the flow cytometer. It should be taken into account that, according to the life cycle of baculovirus, production of GFPVP2 would start around 20 hpi, as its gene is under the very late polh promoter [24]. In contrast, the population expressing gp64, which is expressed in the early and late phases of infection (from 0 to 20-24 hpi), behaved as VP6, which is also under the polh promoter but has a higher production rate than GFPVP2 [3]. Thus, the observed difference cannot be attributed to a difference in infectivity between the two recombinant viruses.

Measurements performed at 24 hpi reflect primary infection. At $48 \mathrm{hpi}$, the population of cells expressing a recombinant protein increased in cultures infected at MOI below $5 \mathrm{pfu} / \mathrm{cell}$. Such an increase was a result of infection by the viral progeny produced during the late phase of the primary infection (12-24 hpi, [24]). At $48 \mathrm{hpi}$, secondary infection resulted in a similar population of cells expressing VP6 regardless of differences in the MOI (Figure 2). In contrast, the population expressing GFPVP2 at MOI below $10 \mathrm{pfu} / \mathrm{cell}$ did not catch up at $48 \mathrm{hpi}$ with that at the higher MOI of 10 or $20 \mathrm{pfu} / \mathrm{cell}$. Such a lower percentage of population expressing GFPVP2 at 48 hpi may be a result of the rapid decline in viability observed in these cultures (Figure 3B), which did not occur in cultures expressing VP6 (data not shown). Wu et al. [28] also observed that infection with baculoviruses containing different recombinant genes results in different cell death kinetics. The decrease in viability was faster as the MOI increased, similarly to what Wu et al. [28] observed in the range of 0.5 to $10 \mathrm{pfu} / \mathrm{cell}$.

In general, population fractions obtained from individually infected cultures cannot be extrapolated to coinfections, a phenomenon that has not been previously described in coinfections with two different recombinant viruses of the same species. The increase of the population expressing VP6 in coinfected cultures may be a result of a cooperative action between both baculoviruses. Cultures infected at low MOI of bacVP6 would result in only a fraction of the population infected with this virus. Progeny virus would begin to bud from $12 \mathrm{hpi}$ [11] and infect cells still susceptible to additional infection. It has been shown that virus binding to infected cells can occur up to $24 \mathrm{hpi}$ [10]. Therefore, cells initially infected with bacGFPVP2 were still susceptible to additional infection by bacVP6, but, in contrast to single infections at low MOI, all the viral proteins and transcription factors of the very late phase of infection would be already present when the VP6 


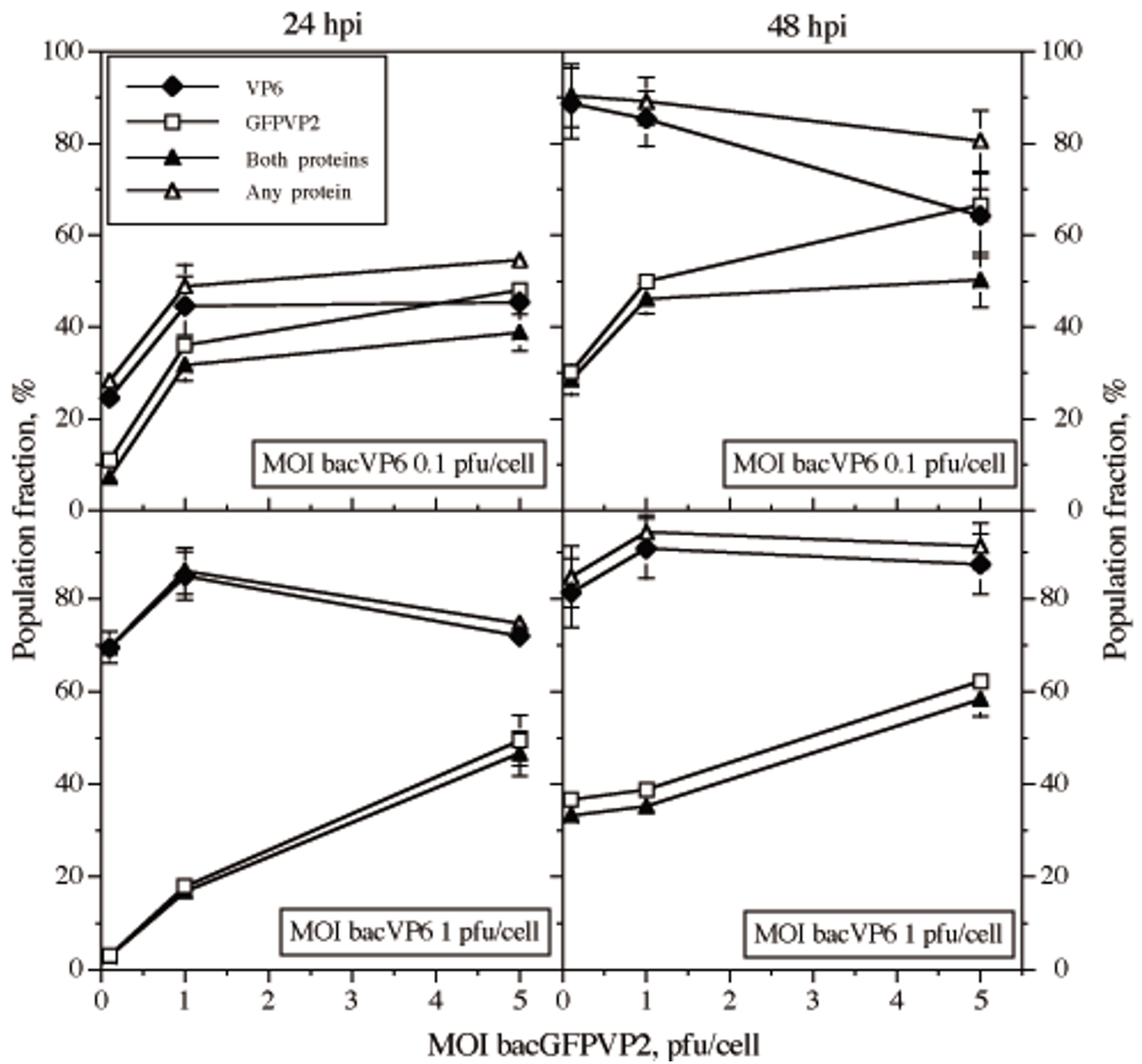

Figure 5

Population expressing GFPVP2, VP6, both recombinant proteins, or any recombinant protein (either GFPVP2 or VP6, or both). Media and difference between duplicate cultures are shown. Some error bars are smaller than the symbols shown.

gene reached the cell nucleus. This may occur as early as 1 hour after progeny virus budding [10]. Accordingly, transcription of the VP6 gene could start, during secondary infection, as early as $13 \mathrm{~h}$ after the initial infection by bacGFPVP2. This would explain the higher population expressing VP6 in coinfections in comparison to individual infections. According to this hypothesis, the difference between the populations expressing VP6 in coinfections or in single infections roughly corresponded to the fraction of cells simultaneously expressing both recombinant proteins. A different situation was observed in the case of the population expressing GFPVP2. At MOI of $5 \mathrm{pfu} / \mathrm{cell}$, the population expressing GFPVP2 decreased around 30\% in coinfections compared to single infections. In general, coinfections at high MOI of GFPVP2 resulted in a lower population expressing a recombinant protein. It appears that secondary infection of bacVP6 interferes with the expression of GFPVP2 by a mechanism still unknown. A similar phenomenon was observed by Alonso et al. [21], in, to our knowledge, the only other work characterizing 


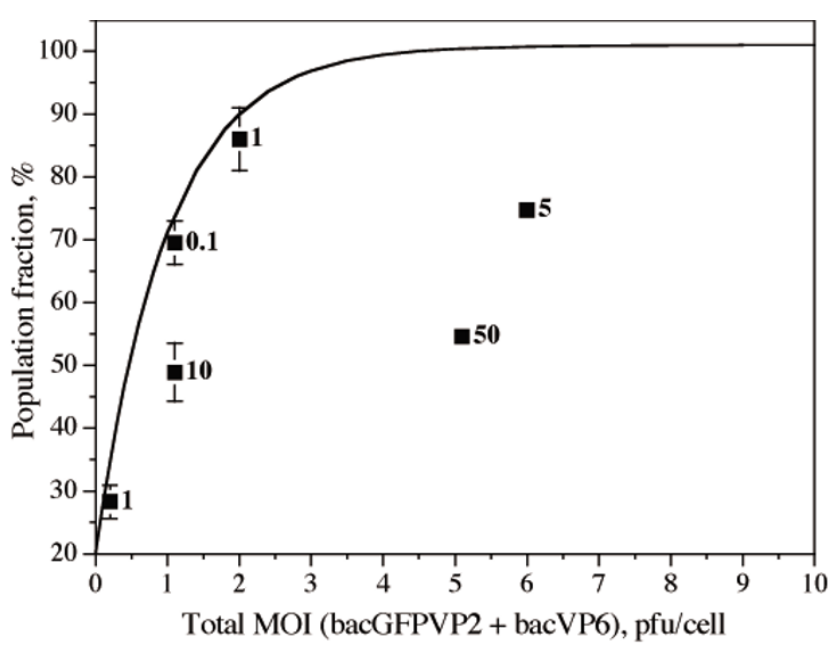

Figure 6

Comparison of coinfected cultures at $\mathbf{2 4}$ hpi with the Poisson distribution. The population fraction expressing any of the recombinant proteins (either GFPVP2 or VP6, or both) is shown. Numbers at the right of symbols are the MOI ratio between bacGFPVP2 and bacVP6.

coinfections with flow cytometry, although using nonrecombinant viruses. In such work, coinfections with hematopoietic necrosis virus and hepatic necrosis virus were screened in a fish cell line. The hepatic necrosis virus interfered with growth of the hematopoietic necrosis virus. However, care should be taken when comparing our work with that of Alonso et al. [21], as they worked with two different virus species. Following mRNA kinetics may help elucidate the interference mechanism of bacVP6 with the expression of GFPVP2. Vieira et al. [4] followed the kinetics of baculovirus replication and found that the number of copies of baculovirus DNA coding for VP2 was about half of that coding for VP6 in coinfected cultures, which could explain a less efficient secondary infection by bacGFPVP2. They also found that no difference exists between the mRNA stability of VP2 or VP6 expressed in the insect-cell baculovirus system. It remains to be determined if the same situation occurs in the case of the fusion gene GFPVP2.

We have previously compared the production of VP2 and VP6 upon infection or coinfection of SF9 cells [3]. We found that the production rates of VP2 and VP6 were similar in individual infections or coinfections at an MOI of $5 \mathrm{pfu} / \mathrm{cell}$. In this work, we did not follow the recombinant protein production rates. However, we did observe that in the culture coinfected with $5 \mathrm{pfu} / \mathrm{cell}$ of bacGFPVP2 and 1 pfu/cell of bacVP6 (the closest conditions to our previous study), the population fraction expressing each of the recombinant proteins was similar to that observed in individually infected cultures. It can be inferred that such cultures would also have a similar recombinant protein production rate, as the cultures we have coinfected in the past at a MOI of 5 pfu.cell.

For efficient production of rotavirus like-particles, adequate VLP assembly would require the simultaneous expression of both GFPVP2 and VP6 by the largest population of cells [8]. Such situation was encountered at a MOI of 5 pfu/cell of bacGFPVP2 and 1 pfu/cell of bacVP6, with $58 \%$ of the population expressing both recombinant proteins at $48 \mathrm{hpi}$. It was observed that the population simultaneously expressing both recombinant proteins closely followed the population expressing GFPVP2, indicating that expression of bacGFPVP2 was the limiting step. We are now working on kinetic studies of the production of single- or double shelled rotavirus-like particles upon infection with bacGFPVP2 and bacVP6.

The characteristics of a recombinant protein and expression system directly affect the production kinetics and determine the limiting steps during its biosynthesis. For instance, molecular weight, posttranslational modifications, the site of accumulation, and other characteristics can affect protein yields. Accordingly, the results presented here can serve as a general practical guideline but inherent characteristics of each particular case must be considered.

\section{Conclusion}

In this work we have, for the first time determined the effect of the simultaneous infection with two recombinant baculovirus on the populations expressing two recombinant proteins. The kinetics of cell populations expressing both recombinant proteins in coinfections could not have been predicted from single infection data or from the Poisson distribution based on theoretical considerations. It was observed that secondary infection of bacVP6 interfered with bacGFP2 expression, and also resulted in a higher percentage of the population expressing VP6. The MOI for each virus that resulted in the highest population percentage expressing both recombinant proteins was found. The information generated in this work describes a novel phenomenon and is useful for designing rational infection strategies needed in several applications for improving the simultaneous expression of two recombinant proteins in systems based on viral gene delivery. Future work in coinfections with several recombinant baculoviruses for producing various proteins by insect cells should take into account that the population behavior of individual infections may be different to that in single infections. 


\section{Methods \\ Cell culture and recombinant protein expression}

High Five cells (Invitrogen, Carlsbad, CA, USA) were cultivated in suspension in SF900-II medium (Invitrogen, Carlsbad, CA, USA) in $250 \mathrm{~mL}$ shaker flasks with $60 \mathrm{~mL}$ of working volume at $27^{\circ} \mathrm{C}$ and $115 \mathrm{rpm}$. Cell concentration and viability prior to infection were determined with a Coulter Counter (Coulter Instruments) and Trypan blue exclusion in a hemacytometer, respectively. Two recombinant baculoviruses derived from Autographa californica nucleopolyhedrovirus (AcMNPV) were used. One baculovirus contained the gene for the fusion protein GFPVP2 (bacGFPVP2, kindly provided by Prof. J. Cohen, INRA, France [29]), and the other contained the gene of VP6 (bacVP6, strain SA11, kindly provided by Dr. S. López, IBT-UNAM, Mexico). Both recombinant genes were under the polh promoter. Viral stocks were titrated as described in Mena et al. [30]. This method has typical standard deviations between 10 and 30\%. Cultures were infected at a cell concentration of $0.5 \times 10^{6}$ cell $\mathrm{mL}^{-1}$ using the different MOI described in the Result section.

\section{Cell preparation for flow cytometry analysis}

To obtain the required cell number for flow cytometry analysis, $15 \mathrm{~mL}$ of culture infected with either bacVP6, bacGFPVP2 or co-infected were centrifuged at 1,000 $\times \mathrm{g}$ for $10 \mathrm{~min}$ in an Eppendorf 5810R centrifuge (Hamburg, Germany) at $4{ }^{\circ} \mathrm{C}$ degrees. The supernatant was discarded and the pellet was washed with $15 \mathrm{~mL}$ of PBS and centrifuged at $1,000 \times \mathrm{g}$ for $10 \mathrm{~min}$. The pellet was then fixed with $15 \mathrm{~mL}$ of $2 \%$ formaldehyde (Sigma-Aldrich, St, Louis, MO, USA) in PBS for $15 \mathrm{~min}$, and washed twice with PBS. Cells were permeabilized for 15 min with $0.2 \%$ sodium deoxycholate (Sigma-Aldrich, St. Louis, MO, USA) in PBS with $2 \%$ bovine serum albumin (SigmaAldrich, St, Louis, MO, USA), and washed twice with PBS. For immunodetection of gp64 and VP6, one mL of pellet was resuspended in $1 \mathrm{~mL}$ of PBS $(0.2 \%$ BSA) with primary antibody in dilution of 1:200 for $1 \mathrm{hr}$. The primary antibodies used were a monoclonal antibody to VP6 (Clone 3C10, Biodesign, Saco, MA, USA), and a monoclonal antibody Fastplax ${ }^{\mathrm{TM}}$ to gp64 (Novagen, Darmstadt, Germany). The cells were washed twice and resuspended in $1 \mathrm{~mL}$ of PBS $(0.2 \%$ BSA) with the secondary antibody at a dilution of 1:200 for $1 \mathrm{~h}$. The secondary antibody was a goat antimouse coupled to R-phycoerythrin (Molecular ProbesInvitrogen, Carlsbad, CA, USA). The cells were washed twice with PBS and resuspended in $500 \mu \mathrm{L}$ of PBS.

\section{Flow cytometry analysis}

A FACSort with CellQuest software (Becton-Dickinson, Franklin Lakes, NJ, USA) was used. The FACSsort has one light source (488 nm) with three-color fluorescence analysis. $100 \mu \mathrm{L}$ of cell preparation was diluted with $400 \mu \mathrm{L}$ of PBS and analyzed in the FACS. Cells expressing GFPVP2 were identified by GFP fluorescence ( $\lambda$ excitation: 488 nm, $\lambda$ emission: 510, FL1 channel). Cells expressing gp64 or VP6 were analyzed by R-phycoerythrin fluorescence $(\lambda$ excitation: $488 \mathrm{~nm}, \lambda$ emission: 575, FL2 channel). In all cases, 10,000 events were analyzed.

\section{Authors' contributions}

JAM performed all the experiments, participated in experiment design and in data analysis and interpretation, and revised the manuscript critically.

OTR participated in experiment design, data analysis and interpretation, critically revised the manuscript, and provided important intellectual content.

LAP participated in the conceptual design of the experiments, in data analysis and interpretation, and drafted the manuscript.

All authors read and approved the final manuscript.

\section{Acknowledgements}

We thank Ana Ruth Pastor, Erika Melchy, and Vanessa Hernández for technical assistance. This work was supported by grants from CONACyTSAGARPA 2004-C0I-103, CONACyT-Morelos MOR-2004-C02-058, and UNAM-PAPIIT IN-223805 and 206407. JAM acknowledges support from DGEP-UNAM during his graduate studies.

\section{References}

I. Palomares LA, Estrada-Mondaca S, Ramírez OT: Principles and applications of the insect-cell-baculovirus expression vector system. In Cell Culture Technology for Pharmaceutical and Cellular Applications Edited by: Ozturk S, Hu WS. New Cork, Taylor and Francis; 2006:627-692.

2. Hu YC, Bentley WE: A kinetic and statistical-thermodynamic model for baculovirus infection and virus-like particle assembly in suspended insect cells. Chem Eng Sci 2002, 55:3991-4008.

3. Palomares LA, López S, Ramírez OT: Strategies for manipulating the relative concentration of recombinant rotavirus structural proteins during simultaneous production by insect cells. Biotechnol Bioeng 2002, 78:635-644.

4. Vieira HLA, Estevao C, Roldao A, Peixoto CC, Sousa MFQ, Cruz PE, Carrondo MJT, Alves PM: Triple layered rotavirus VLP production: Kinetics of vector replication, mRNA stability and recombinant protein production. J Biotechnol 2005, I 20:72-82.

5. Hu YC, Bentley WE: Effect of MOI ratio on the composition and yield of chimeric infectious bursal disease virus-like particles by baculovirus co-infection: Deterministic predictions and experimental results. Biotechnol Bioeng 200 I, 75: I04-II9.

6. Tsao E, Mason M, Cacciuttolo M, Bowen S: Production of parvovirus $B \mid 9$ vaccine in insect cells coinfected with double baculovirus. Biotechnol Bioeng 1996, 49:130-138.

7. Crawford SE, Labbé M, Cohen J, Burroughs M, Zhou Y, Estes MK: Characterization of virus-like particles produced by the expression of rotavirus capsid proteins by insect cells. J Virol 1994, 68:5945-5952.

8. Mena JA, Ramírez OT, Palomares LA: Intracellular distribution of rotavirus structural proteins and virus-like particles expressed in the insect cell-baculovirus system. J Biotechnol 2006, I 22:443-452.

9. Urabe M, Ding C, Kotin RM: Insect cells as a factory to produce Adeno-Associated virus type 2 vectors. Human Gene Ther 2002, I3:1935-1943.

10. Dee KU, Shuler ML: A mathematical model of the trafficking of acid-dependent enveloped viruses: Application to the bind- 
ing, uptake, and nuclear accumulation of baculovirus. Biotechnol Bioeng 1997, 54:468-490.

II. Enden G, Zhang YH, Merchuk JC: A model of the dynamics of insect cell infection at low multiplicity of infection. J Theoretical Biol 2005, 237:257-264.

12. Gotoh T, Chiba K, Kikuchi K: Probabilistic characterization for baculovirus-infected insect cells destined to synthesize progeny viruses and recombinant protein and to die. J Chem Eng Japan 2004, 37: I357-1366.

13. Licari P, Bailey J: Modeling the population-dynamics of baculovirus-infected cells-optimizing infection strategies for enhanced recombinant protein yields. Biotechnol Bioeng 1992, 39:432-44I.

14. Roldao A, Vieira HLA, Alves PM, Olieira R, Carrondo MJT: Intracellular dynamics in rotavirus-like particles production: Evaluation of multigene and monocistronic infection strategies. Process Biochem 2006, 4I:2188-2199.

15. Deparis V, Jestin A, Marc A, Giergen JL: Use of flow cytometry to monitor infection and recombinant human $\alpha-I, 3 / 4$ fucosyltransferase production in baculovirus infected Sf9 cell cultures. Biotechnol Prog 2003, 19:624-630.

16. Eriksson S, Raivio E, Kukkonen JP, Eriksson K, Lindqvist C: Green fluorescent protein as a tool for screening recombinant baculoviruses. J Virological Meth 1996, 59:127-133.

17. Morris TD, Miller LK: Characterization of productive and nonproductive ACMNPV infection in selected insect cell lines. Virol 1993, 197:339-348.

18. Schopf B, Howaldt MW, Bailey JE: DNA distribution and respiratory activity of Spodoptera frugiperda populations infected with wild-type and recombinant Autographa californica nuclear polyhedrosis virus. J Biotechnol 1990, I5:169-186.

19. Gueret V, Negrete-Virgen JA, Lyddiatt A, Al-Rubeai M: Rapid titration of adenoviral infectivity by flow cytometry in batch culture of infected HEK293 cells. Cytotechnol 2002, 38:87-97.

20. Chan ZR, Lai CW, Lee HP, Chen HC, Hu YC: Determination of the baculovirus transducing titer in mammalian cells. Biotechnol Bioeng 2006, 93:564-57I.

21. Alonso M, Rodríguez S, Pérez-Prieto SI: Viral coinfection in salmonids: infectious pancreatic necrotic virus interferes with infectious hematopoietic necrosis virus. Arch Virol 1999, 144:657-673.

22. Kitajima M, Hamazaki H, Miyano-Kurosaki N, Takaku H: Characterization of baculovirus Autographa californica multiple nuclear polyhedrosis virus infection in mammalian cells. Biochem Biophys Res Com 2006, 343:378-384.

23. Whitford M, Stewart S, Kuzio J, Faulkner P: Identification and sequence analysis of a gene encoding gp67, an abundant glycoprotein of the baculovirus Autographa californica nuclear polyhedrosis virus. J Virol 1989, 63:1393-1399.

24. O'Reilly DR, Miller LK, Luckow VA: Baculovirus Expression Vectors. A laboratory manual New York, Oxford University Press; 1994.

25. Bleckwenn NA, Bentley WE, Shiloach J: Evaluation of production parameters with the vaccinia virus expression system using microcarrier attached HeLa cells. Biotechnol Prog 2005, 21:554-56I.

26. Chillakuru RA, Ryu DDY, Yilma T: Propagation of recombinant vaccinia virus in HeLa cells: Adsoprtion kinetics and replication in batch cultures. Biotechnol Prog |99|, 7:85-92.

27. Petricevich VL, Palomares LA, González M, Ramírez OT: Parameters that determine virus adsorption kinetics: Toward the design of better infection strategies for the insect cell - baculovirus expression system. Enzyme Microb Technol 200I, 29:52-6I.

28. Wu S-C, Jarvis DL, Dale BE, Liao JC: Heterologous protein expression affects the death kinetics of baculovirus-infected insect cell cultures: $A$ quantitative study by use of $n$-target theory. Biotechnol Prog 1994, 10:55-59.

29. Charpilienne A, Nejmeddine M, Berois M, Parez N, Neumann E, Hewat E, Trugnan G, Cohen J: Individual rotavirus-like particles containing I 20 molecules of fluorescent protein are visible in living cells. J Biol Chem 200I, 276:2936I-29367.

30. Mena JA, Ramírez OT, Palomares LA: Titration of non-occluded baculovirus using a cell viability assay. BioTechniques 2003 , 34:260-264.
Publish with Biomed Central and every scientist can read your work free of charge

"BioMed Central will be the most significant development for disseminating the results of biomedical research in our lifetime. "

Sir Paul Nurse, Cancer Research UK

Your research papers will be:

- available free of charge to the entire biomedical community

- peer reviewed and published immediately upon acceptance

- cited in PubMed and archived on PubMed Central

- yours - you keep the copyright 